\title{
Pharmacological stimulation of p53 with low-dose doxorubicin ameliorates diet-induced nonalcoholic steatosis and steatohepatitis
}

\author{
Begoña Porteiro ${ }^{1,2}$, Marcos F. Fondevila ${ }^{1,2}$, Xabier Buque ${ }^{3,4}$, Maria J. Gonzalez-Rellan ${ }^{1,2}$, Uxia Fernandez ${ }^{1,2}$, \\ Alfonso Mora ${ }^{5}$, Daniel Beiroa ${ }^{1,2}$, Ana Senra ${ }^{1}$, Rosalia Gallego ${ }^{6}$, Johan Ferno ${ }^{7,8}$, Miguel López ${ }^{1,2}$, \\ Guadalupe Sabio ${ }^{5}$, Carlos Dieguez ${ }^{1,2}$, Patricia Aspichueta ${ }^{3,4}$, Rubén Nogueiras ${ }^{1,2, *}$
}

ABSTRACT

Objective: Recent reports have implicated the p53 tumor suppressor in the regulation of lipid metabolism. We hypothesized that the pharmacological activation of p53 with low-dose doxorubicin, which is widely used to treat several types of cancer, may have beneficial effects on nonalcoholic fatty liver disease (NAFLD) and nonalcoholic steatohepatitis (NASH).

Methods: We used long-term pharmacological activation of p53 by i.p. or oral administration of low-dose doxorubicin in different animal models of NAFLD (high fat diet containing $45 \%$ and $60 \%$ kcal fat) and NASH (methionine- and choline-deficient diet and choline deficiency combined with high fat diet). We also administered doxorubicin in mice lacking p53 in the liver and in two human hepatic cells lines (HepG2 and THLE2). Results: The attenuation of liver damage was accompanied by the stimulation of fatty acid oxidation and decrease of lipogenesis, inflammation, and ER stress. The effects of doxorubicin were abrogated in mice with liver-specific ablation of p53. Finally, the effects of doxorubicin on lipid metabolism found in animal models were also present in two human hepatic cells lines, in which the drug stimulated fatty acid oxidation and inhibited de novo lipogenesis at doses that did not cause changes in apoptosis or cell viability.

Conclusion: These data provide new evidence for targeting p53 as a strategy to treat liver disease.

(c) 2017 The Authors. Published by Elsevier GmbH. This is an open access article under the CC BY-NC-ND license (http://creativecommons.org/licenses/by-nc-nd/4.0/).

Keywords Obesity; Lipid metabolism; Inflammation

\section{INTRODUCTION}

p53 is an intensively studied protein, primarily as a tumor suppressor in humans and other mammals [1]. p53 mutations or deficiency are highly correlated with increased susceptibility to cancer, and most studies have focused on how p53 might protect against malignant progression. Besides cell proliferation, p53 plays an important role in biological functions such as stress, ageing, and obesity-associated disorders [2].
Although there is a large body of evidence showing that p53 promotes fatty acid catabolism while it inhibits anabolism through the regulation of gene expression [3], the possible contribution of p53 to the pathogenesis of nonalcoholic fatty liver disease (NAFLD) has only recently begun to be understood. Manipulations of p53 specifically in the liver, thus avoiding possible contributions/compensations from p53 modulations in other tissues, found that the lack of hepatic p53 increases lipid deposition while its over-expression ameliorates high fat dietinduced steatosis $[4,5]$. These latter results are also in agreement

\footnotetext{
${ }^{1}$ Department of Physiology, CIMUS, University of Santiago de Compostela-Instituto de Investigación Sanitaria, Santiago de Compostela, 15782, Spain ${ }^{2}$ CIBER Fisiopatología de la Obesidad y Nutrición (CIBERobn), 15706, Spain ${ }^{3}$ Department of Physiology, Faculty of Medicine and Nursing, University of the Basque Country UPV/EHU, Spain ${ }^{4}$ Biocruces Research Institute, Spain ${ }^{5}$ Centro Nacional de Investigaciones Cardiovasculares Carlos III (CNIC), Madrid, Spain ${ }^{6}$ Department of Morphological Sciences, School of Medicine, University of Santiago de Compostela-Instituto de Investigación Sanitaria, Santiago de Compostela, 15782, Spain ${ }^{7}$ KG Jebsen Center for Diabetes Research, Department of Clinical Science, University of Bergen, Bergen, Norway ${ }^{8}$ Hormone Laboratory, Haukeland University Hospital, Bergen, Norway

*Corresponding author. Department of Physiology, CIMUS, University of Santiago de Compostela \& CIBER Fisiopatología de la Obesidad y Nutrición (CIBERobn); Avenida de Barcelona s/n, 15782 Santiago de Compostela (A Coruña), Spain. E-mail: ruben.nogueiras@usc.es (R. Nogueiras).

\begin{abstract}
Abbreviations: AAV8, adeno-associated virus serotype 8; Abcd1, ATP binding cassette subfamily D member 1; Acadl, acyl-CoA deshydrogenase, long-chain; Acadm, acylCoA deshydrogenase, medium chain; ACC, acetyl-CoA carboxylase; Acox, acyl-CoA oxidase 1; Ad, adenovirus; ALT, alanine aminotransferase; ApoB, apolipoprotein b; ASM, acid-soluble metabolites; AST, aspartate aminotransferase; AUC, area under curve; BSA, bovine serum albumin; CDHFD, choline-deficient high fat diet; CHOP, C/EBPhomologous protein; DG, diacylglycerol; DI0, diet-induced obesity; DN, dominant negative; DOX, Doxorubicin; EC, sterified cholesterol; ER stress, endoplasmic reticulum stress; FAS, fatty acid synthase; Fatp2, solute carrier family 27 (fatty acid transporter) member 2; FA, free fatty acid; GAPDH, glyceraldehyde-3-phosphate deshydrogenase; GFP, green fluorescent protein; GTT, glucose test tolerance; HFD, high fat diet; i.p., intraperitoneal; IL10, interleukin 10; IL1 $\beta$, interleukin 1beta; IL6, initerleukin 6; ITT, insulin test tolerance; JNK, C-Jun N-terminal kinase; MCD, methionine-choline deficient diet; NAFLD, non-alcoholic fatty liver disease; NASH, non-alcoholic steatohepatitis; $\mathrm{NF} \kappa \beta$, nuclear factor kappa b; OA, oleic acid; pACC, phospho acetyl-CoA carboxylase; pIRE, Inositol-requiring enzyme $1 \alpha$; pJNK, phospho c-Jun N-terminal kinase; Ppar $\alpha$, peroxisome proliferator activated receptor alpha; Quer, quercetin; SPF, specific pathogen free room; TG, triglyceride; TNF $\alpha$, tumor necrosis factor alpha; UPR, unfolded protein response; XBP1s, X-box binding protein 1
\end{abstract}

Received December 5, 2017 • Accepted December 12, 2017 • Available online 15 December 2017

https://doi.org/10.1016/j.molmet.2017.12.005 
with in vitro studies suggesting a catabolic action of p53 on fatty acids $[3,6]$.

The anthracycline doxorubicin is an important chemotherapeutic agent used in the treatment of solid epithelial and mesenchymal tumors as well as leukemias. p53 is essential for the therapeutic action of doxorubicin since p53 mutations are associated with resistance to this drug [7]. Therefore, we tested the hypothesis that doxorubicin treatment at much lower doses than those used for chemotherapy may have beneficial effects in animal models of NAFLD and nonalcoholic steatohepatitis (NASH). Importantly, we assessed the feasibility of chronic treatment with oral administration of doxorubicin in these preclinical models. The interest in this approach was further prompted by our recent data showing that doxorubicin exerted a beneficial effect on body weight [8].

In the present study, we demonstrate that chronic pharmacological stimulation of p53 with a low dose of doxorubicin (administered intraperitoneally and orally) improves liver injury in different models of diet-induced steatosis and NASH through stimulation of fatty acid oxidation and decrease of lipogenesis, inflammation, and ER stress. These effects are unlikely to be off-target effects of doxorubicin, since they did not occur when the drug was administered to mice with liverspecific ablation of p53. Finally, the effects of doxorubicin on lipid metabolism found in animal models were also present in human hepatic cells lines. These data provide new evidence for p53 targeting as a promising strategy for liver disease.

\section{MATERIAL AND METHODS}

\subsection{Animals and diets}

Animal experiments were conducted in accordance to the standards approved by the Faculty Animal Committee at the University of Santiago de Compostela, and the experiments were performed in agreement with the Rules of Laboratory Animal Care and International Law on Animal Experimentation. 8-10 weeks old Swiss mice were kept under a $12 \mathrm{~h}$ light/dark cycle and had ad libitum access to chow diet and water. In the first study, we used Swiss mice (8-10 weeks old) kept under a $12 \mathrm{~h}$ light/dark cycle and with ad libitum access to chow diet and water mice fed a HFD (Research Diets D12451; 45\% fat, $4.73 \mathrm{kcal} / \mathrm{g}$ ) for 3 months before undergoing the treatments. In a second study, we used C57BL/6J mice (8-10 weeks old) fed a HFD (Research Diets, D12492; 60\% fat, $5.24 \mathrm{kcal} / \mathrm{g}$ ) for 2 months, MCD diet (D05010402, Research Diets) for 2 weeks and CD-HFD (A0282002B, Research diets) for 4 weeks before undergoing the treatments. p53 floxed mice were purchased from The Jackson Laboratory (B6.129P2-Trp53tm1Brn/J) and maintained on a Specific Pathogen Free room (SPF) and fed with chow diet or HFD (Research Diets, D12492; $60 \%$ fat, $5.24 \mathrm{kcal} / \mathrm{g}$ ) for two months before undergoing the treatments. Food intake and body weight were measured twice a week during experimental phase in all experiments.

\subsection{Intraperitoneal (i.p.) injections}

For acute i.p. injections, Doxorubicin hydrochloride (Tocris) was dissolved in saline, which was used as a vehicle. Doxorubicin was administered at $0.15,0.3,0.6,1.25$, and $2.5 \mathrm{mg} / \mathrm{kg}$.

\subsection{Oral gavage administration}

C57BL/6J mice fed with MCD and CDHFD were orally administrated doxorubicin 10 and $20 \mathrm{mg} / \mathrm{kg}$ twice a week for 2 weeks. In this procedure, a bulb tipped gastric gavage was attached to a syringe and used to deliver doxorubicin directly into the stomach (BIOSEB). $30 \mathrm{~min}$ before doxorubicin administration, mice received oral quercetin $15 \mathrm{mg} /$ $\mathrm{kg}$ since it is known that enhance the bioavailability of orally administrated doxorubicin in the intestine [9].

\subsection{In vivo adenoviral gene transfer}

For the down-regulation of p53 specifically in the liver, p53 floxed mice were injected in the tail vein $\left(1 \times 10^{10} \mathrm{VG} / \mathrm{mL}\right)$ with an AAV8 encoding Cre recombinase (SL100835 Signagen Laboratories) or GFP (SL100833 Signagen Laboratories) [5]. In addition, we also used C57BL/6J mice treated with adenoviral vectors encoding GFP alone (Ad-GFP) or the double-mutant kinase dead p53 regulating kinase (DNTp53RK) acting as a dominant negative (the Tp53RK clone was then mutated at g217a and a218t to E73M) that block p53 activation and function (Ad-DNTp53RK) (Signagen, reference numbers SL100708 and SL115889 respectively) [8].

\subsection{Determination of body composition}

Whole body composition was measured using nuclear magnetic resonance (NMR) imaging (Whole Body Composition Analyzer; EchoMRI).

\subsection{RNA isolation and quantitative real-time PCR (qRT-PCR) analysis}

Total liver RNA was extracted and qRT-PCR was performed as previously described [5] (see Supplementary information and Supplementary Table 3 for primers used).

\subsection{Western blot analysis}

Western blot was performed as described [5] (see Supplementary information and Supplementary Table 4 for antibodies used).

\subsection{Cell culture and transfection}

THLE2 and HepG2 cells were cultured and transfected as previously described [5] (see Supplementary information).

\subsection{De novo lipogenesis and fatty acid oxidation}

De novo lipogenesis and the rate of fatty acid oxidation were determined as previously described [5] (see Supplementary information).

2.10. Data analysis and statistics

A Kolgomorov-Smirnov test $(n=5-7)$ and the Shapiro-Wilk test ( $\mathrm{n} \geq 7$ ) were performed to test normality. When variables were normally distributed, Student's $t$-test (for comparisons between two groups) or analysis of variance followed by post hoc Bonferroni adjustment were performed. When variables were not normally distributed, we performed the non-parametric Mann-Whitney U-test (for comparisons between two groups) or a Kruskal-Wallis test, followed by Dunn's multiple comparison test, when more than two groups were compared. Tests were performed with PRISM (GraphPad Software v5). Data are presented as mean \pm s.e.m. and statistical significance was set at $p<0.05$ for all analyses.

\section{RESULTS}

3.1. Intraperitoneal doxorubicin suppresses body weight gain and ameliorates liver steatosis in diet-induced obese mice in a feedingindependent manner

We first injected four different doses of intraperitoneal (i.p.) doxorubicin $(0.3,0.6$ and 1.25 and $2.5 \mathrm{mg} / \mathrm{kg}$, daily for 5 days) in mice fed a chow diet. We found that after 5 days of treatment, the highest dose $(2.5 \mathrm{mg} /$ $\mathrm{kg}$ ) decreased body weight, adiposity, and food intake while the doses of 0.6 and $1.25 \mathrm{mg} / \mathrm{kg}$ decreased weight gain and adiposity in a 
feeding-independent manner (Supplementary figures $1 \mathrm{~A}-\mathrm{C}$ ). The lowest dose $(0.3 \mathrm{mg} / \mathrm{kg})$ did not change body weight relative to vehicle, despite a small statistically significant increase in food intake.

Next, we investigated the effect of different doses of doxorubicin (i.p injection of $0.15,0.3,0.6$ and $1.25 \mathrm{mg} / \mathrm{kg}, 3$ times/week for 2 months) in diet-induced obese (DI0) Swiss mice, with a diet consisting of $45 \%$ fat. All the doses of doxorubicin reduced weight gain during 2 months of treatment (Figure $1 \mathrm{~A}$ ). This effect occurred without any significant changes in food intake, except for the highest dose $(1.25 \mathrm{mg} / \mathrm{kg})$ at which food intake was decreased (Figure 1A). The dose of $0.6 \mathrm{mg} / \mathrm{kg}$ caused the most pronounced weight loss in a feeding-independent manner and was sufficient to phosphorylate p53 and to up-regulate the p53 downstream molecule Bax (Figure 1B), so we chose this dose for further analyses. At this dose, doxorubicin decreased fat mass without changes in non-fat mass (Figure 1C), reduced the hepatic triglyceride content, and decreased serum levels of AST and ALT (Figure 1D). Serum troponin levels, measured as a marker of myocardial injury, did not show differences between vehicle and doxorubicin treated mice (Supplementary Table 1). A decrease in serum triglycerides and free fatty acids (FFA) was detected after doxorubicin treatment (Supplementary Table 2). To investigate the potential mechanisms affected by doxorubicin, we measured markers of several pathways that are widely known to be altered in murine models of NAFLD, such as markers of lipid oxidation and export (Acox, Acadm, Acadl, Abcd1, Fatp2, Ppara, and ApoB), unfolded protein response (UPR) (pIRE, XBP1s, and CHOP), pJNK, and inflammation (IL6, IL10, NFK $\beta$, Tnf $\alpha$, arginase, and F480). Our data showed that doxorubicin stimulated the gene expression of several markers of lipid oxidation, such as Acox, Acadm, and Fatp2, and decreased the expression of markers for endoplasmic reticulum (ER) stress and inflammation in comparison to vehicle-treated mice (Figure 1Eand F).

In order to investigate whether the beneficial effect of doxorubicin that was observed in Swiss mice fed a high fat diet (HFD with $45 \%$ of fat) also occurred in another DIO animal model, we next followed the same experimental protocol (i.p. doxorubicin $0.15,0.3$ and $0.6 \mathrm{mg} /$ $\mathrm{kg}, 3$ times/week for 2 months) in C57BL/6J mice on a more severe diet (HFD with $60 \%$ of fat). The reason for using a distinct strain was to demonstrate that the effects of doxorubicin are consistent in different models. We found that after 2 months, the doses of 0.3 and $0.6 \mathrm{mg} / \mathrm{kg}$ reduced weight gain in a feeding-independent manner (Figure 2A), with only a marginal decrease in food intake in animals treated with the highest dose at the last week (Figure 2A). Consistent with the reduced weight gain, fat mass was also decreased at these 2 doses, while non-fat mass decreased only after the treatment with the dose of $0.6 \mathrm{mg} / \mathrm{kg}$ (Figure 2B). Doxorubicin decreased hepatic triglyceride content (Figure $2 \mathrm{C}$ and D), serum AST and ALT (Figure 2D), with no changes in serum troponin levels (Supplementary Table 1) or in serum triglycerides (Supplementary Table 2). The doses of 0.3 and $0.6 \mathrm{mg} / \mathrm{kg}$ were sufficient to increase phosphorylated levels of p53 and Bax (Figure 2E). Of note, the chronic treatment with doxorubicin at 0.3 and $0.6 \mathrm{mg} / \mathrm{kg}$ improved glucose tolerance without affecting insulin sensitivity (Figure 2F). However, a single injection of doxorubicin did not alter glucose tolerance after $24 \mathrm{~h}$ in DIO mice (Figure 2F).

\subsection{Oral doxorubicin ameliorates liver damage in diet-induced NAFLD and NASH without affecting body weight}

Since our findings indicated that the chronic i.p. treatment with doxorubicin had beneficial effects in terms of liver steatosis in DIO mice, we tested the feasibility of developing an oral treatment. Indeed, the bioavailability of oral doxorubicin is very low but previous reports found that the isoflavone quercetin increased its bioavailability [9]. Thus, we treated DIO mice with quercetin and two different doses of doxorubicin (10 and $20 \mathrm{mg} / \mathrm{kg}$ ) for 7 weeks. We found that while the dose of $10 \mathrm{mg} / \mathrm{kg}$ was ineffective, the dose of $20 \mathrm{mg} / \mathrm{kg}$ decreased body weight increment and adiposity independently of feeding (Supplementary Figures $2 \mathrm{~A}-\mathrm{C}$ ), and decreased hepatic triglyceride levels and serum AST (Supplementary Figures 2D-E). A single administration of oral doxorubicin $(20 \mathrm{mg} / \mathrm{kg})$ did not alter glucose tolerance after $24 \mathrm{~h}$ in DIO mice (Supplementary Figure 2F).

Once we found that the oral treatment of doxorubicin was effective to ameliorate liver steatosis in DIO mice, we next assessed its function in a more advanced stage of the disease. In order to evaluate this, we used mice fed a methionine- and choline-deficient (MCD) diet, which develop NASH and fibrosis [10]. We found that the cotreatment of quercetin with doxorubicin $(20 \mathrm{mg} / \mathrm{kg})$ did not affect body weight in comparison to vehicle treated mice (Figure $3 \mathrm{~A}$ ), but it decreased ballooning, the concentration of hepatic triglycerides, and serum AST and ALT levels (Figure $3 \mathrm{~B}$ ), while serum troponin levels, triglycerides and free fatty acids (FFA) remained unchanged (Supplementary Tables 1 and 4). Mice treated orally with doxorubicin also showed lower protein levels and gene expression of collagen III (Figure 3C), suggesting a reduction in fibrosis. Oral doxorubicin activated the p53 signaling pathway since it stimulated protein levels of p21 and Bax (Figure 3D). In line with this, treated mice showed increased hepatic expression of several major markers of lipid oxidation and transport (Figure 3E), and decreased levels of markers of lipogenesis, ER stress as well as inflammation (Figure 3F).

To further evaluate doxorubicin treatment in an animal model of liver damage more similar to that observed in humans, mice were fed a diet combining choline deficiency with HFD (CD-HFD) [11,12]. Mice treated with oral doxorubicin did not show differences in body weight when compared to vehicle-treated mice (Figure 4A). However, ballooning, the levels of hepatic triglycerides and serum AST and ALT levels were significantly decreased in mice receiving doxorubicin (Figure 4B), with no changes in serum troponin levels, triglycerides, and free fatty acids (FFA) (Supplementary Tables 1 and 4). This treatment also diminished protein levels and gene expression of collagen III (Figure 4C). Oral doxorubicin increased protein levels of phosphorylated p53 and Bax (Figure 4D). In addition, doxorubicin-treated mice showed increased hepatic expression of several markers of lipolysis (Figure $4 \mathrm{E}$ ), and decreased levels of markers of lipogenesis, ER stress as well as inflammation (Figure 4F).

\subsection{Doxorubicin does not ameliorate liver steatosis in mice with} hepatic knockdown of p53

To investigate whether the hepatic actions of doxorubicin are specifically mediated by $\mathrm{p53}$, we studied the drug effects in mice with p53 knocked down specifically in their liver using dominant negative (DN) virus. The efficacy of the virus was proved by significantly downregulated p53 expression (Figure $5 \mathrm{~A}$ ). As expected [4,5], these mice displayed increased lipid deposition in the liver (Figure 5B). However, $0.6 \mathrm{mg} / \mathrm{kg}$ doxorubicin administered i.p. daily for 3 weeks did not affect hepatic lipid content (Figure $5 \mathrm{~B}$ ). Consistent with these results, the expression of markers of lipogenesis and ER stress were elevated in the p53 knockout mice, whereas doxorubicin was unable to reverse these effects (Figure 5C).

In order to verify these results in another knockdown model, we also deleted hepatic p53 using the cre-lox recombination. Thus, p53 floxed mice were injected in the tail vein with AAV8 expressing GFP alone (controls) or GFP combined with Cre. The efficacy of the recombination 


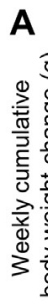

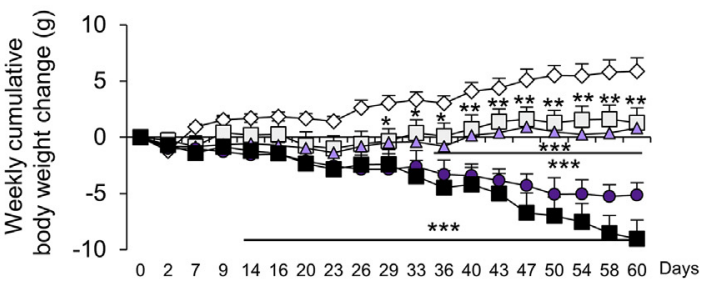

B

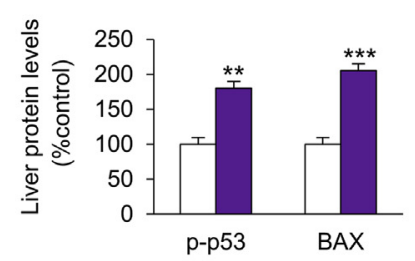

$\square$ Vehicle

$\square$ DOX $0.6 \mathrm{mg} / \mathrm{Kg}$

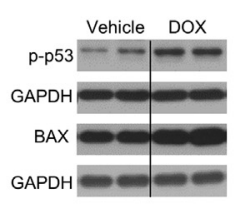

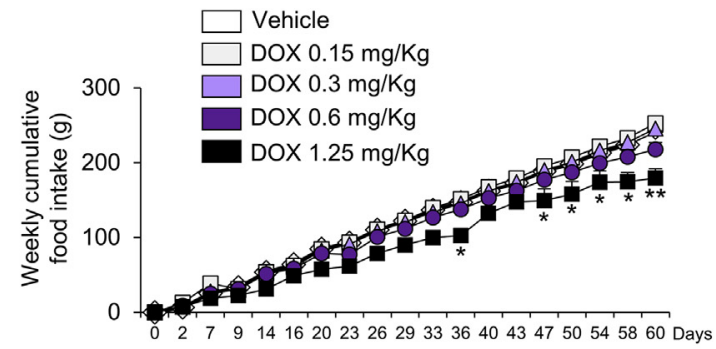

C

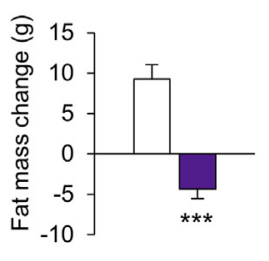

D
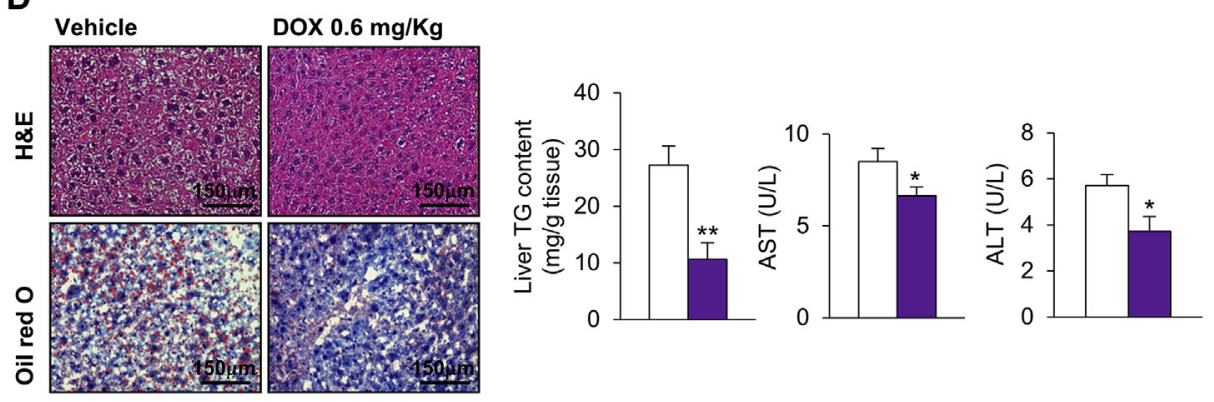

E

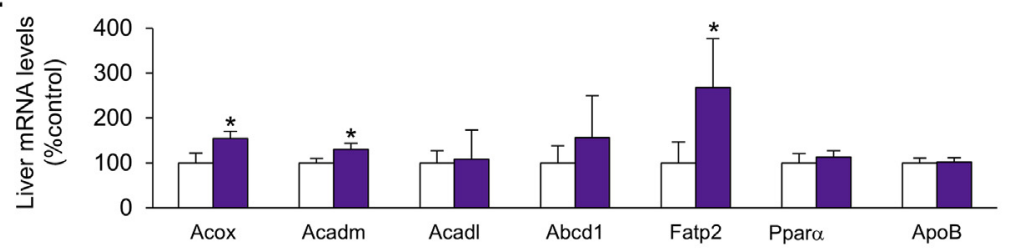

F
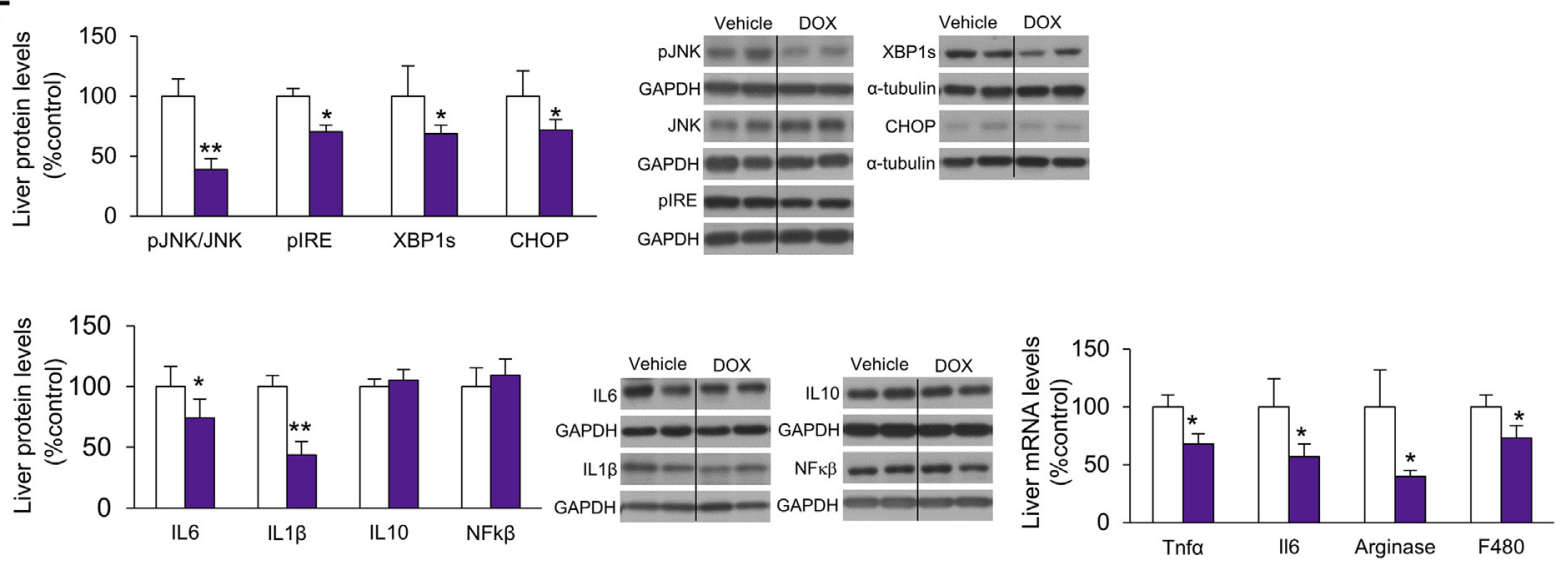

Figure 1: Metabolic effects of doxorubicin treatment in obese Swiss mice. Swiss mice fed a HFD (45\% fat) for 12 weeks, were randomly treated with either saline or doxorubicin at different doses injected intraperitoneally twice a week during next 60 days. Cumulative body weight and food intake (A), phosphorylated levels of p53 and Bax (B), and changes in body composition between control and $0.6 \mathrm{mg} / \mathrm{kg}$ doxorubicin treated mice (C). Representative photomicrographs of hematoxylin\&eosin (upper panel) and oil red 0 staining (lower panel) of liver sections, liver TG content, and serum levels of AST and ALT (D). mRNA levels of genes related to fatty acid oxidation in liver samples (E). Liver protein levels of proteins involved in ER stress and gene expression of inflammatory markers (F). HPRT, tubulin, and GAPDH were used to normalize mRNA and protein levels. Dividing lines indicate splicings in the same gel. Values are mean \pm standard error of the mean of $7-10$ animals per group. ${ }^{\star} p<0.05$, ${ }^{\star \star} p<0.01,{ }^{\star \star \star} p<0.001$ versus control group. 
A
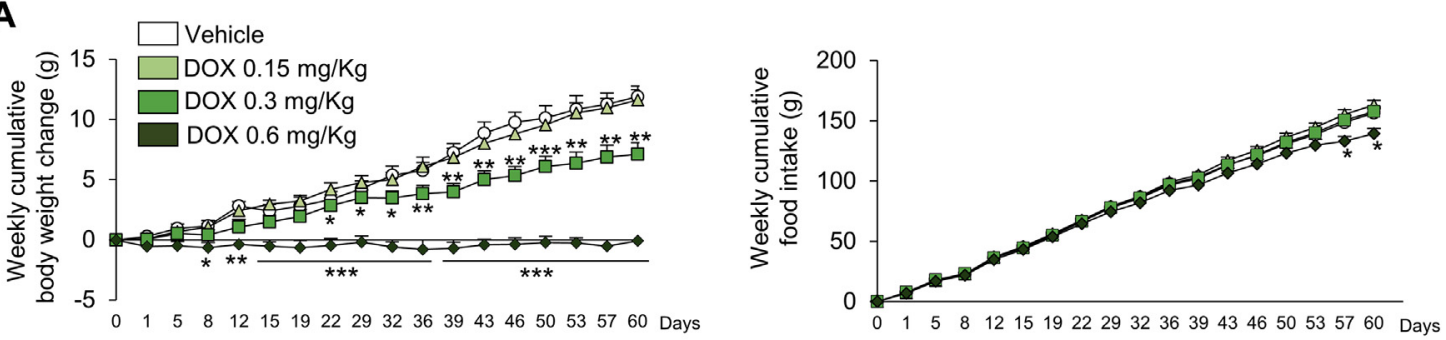

B

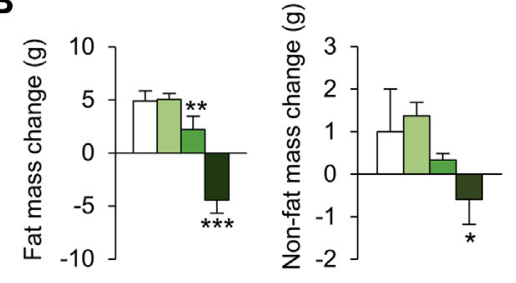

C

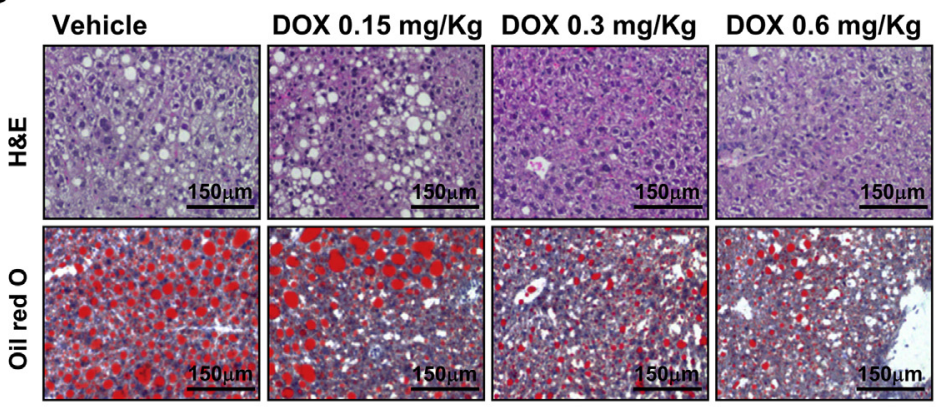

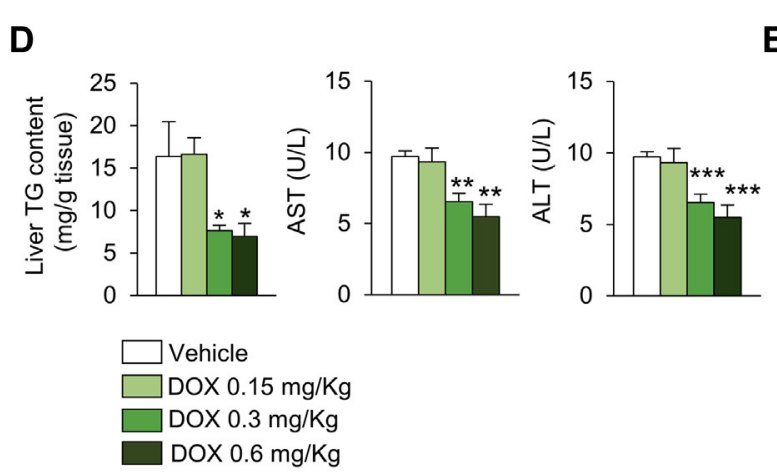

E
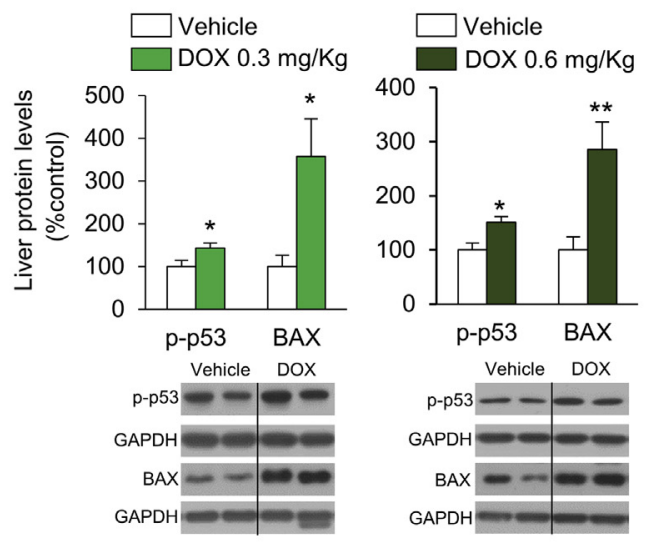

F
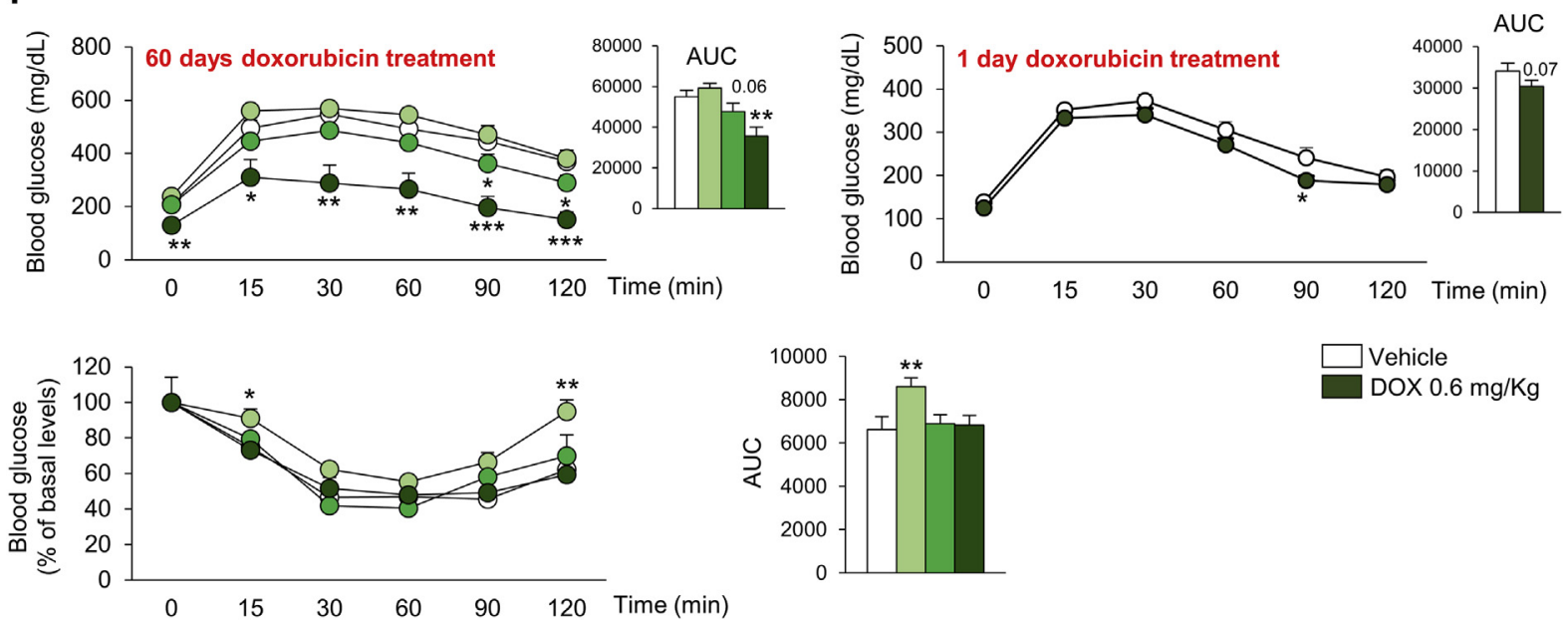

Vehicle

DOX $0.6 \mathrm{mg} / \mathrm{Kg}$

Figure 2: Metabolic effects of doxorubicin treatment in C57BL/6J obese mice. Mice fed a HFD (60\% fat) for 8 weeks were randomly treated with either saline or doxorubicin at different doses injected intraperitoneally twice a week during next 60 days. Cumulative body weight and food intake (A) and changes in fat mass and non-fat mass (B) between control and $0.6 \mathrm{mg} / \mathrm{kg}$ doxorubicin treated mice. Representative photomicrographs of hematoxylin\&eosin (upper panel) and oil red 0 staining (lower panel) of liver sections (C). Liver TG content and serum levels of AST and ALT (D). Phosphorylated levels of p53 and Bax in control and doxorubicin (0.3 and $0.6 \mathrm{mg} / \mathrm{kg})$ treated mice (E). Blood glucose levels during intraperitoneal GTT $(2 \mathrm{~g} / \mathrm{kg})$ after chronic or acute treatment with doxorubicin and ITT $(0,75 \mathrm{U} / \mathrm{kg})$ in $6 \mathrm{~h}$ fasted mice after doxorubicin treatment and the corresponding areas under curve (F). Values are mean \pm standard error of the mean of $7-10$ animals per group. ${ }^{\star} p<0.05,{ }^{\star \star} p<0.01$, ${ }^{\star \star \star} p<0.001$ versus control group. 
A

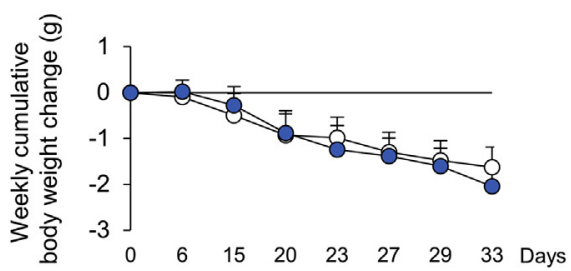

B

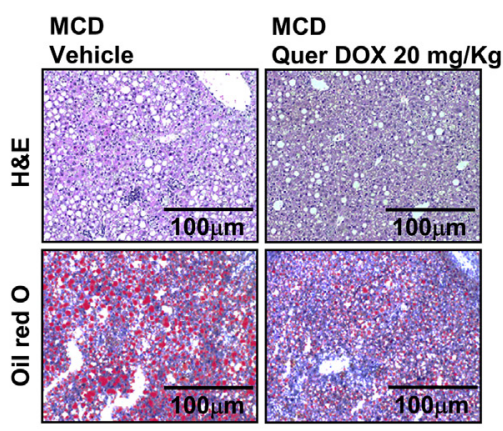

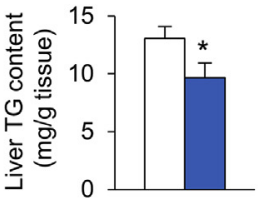

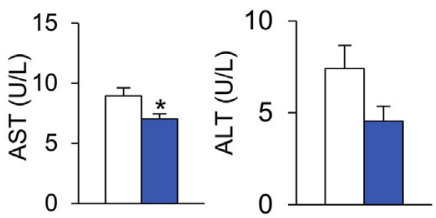

C

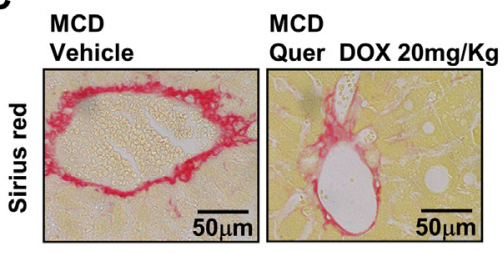

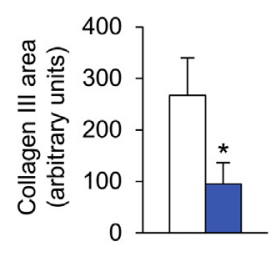

D

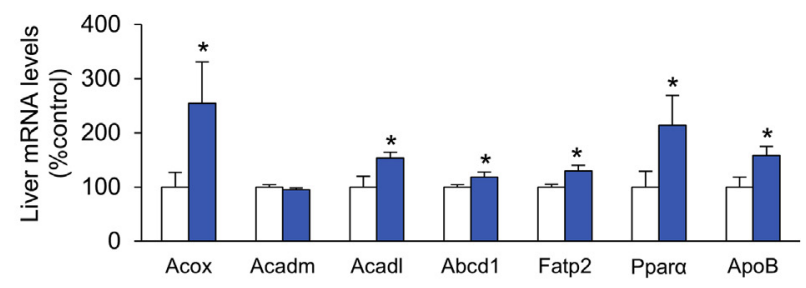

E

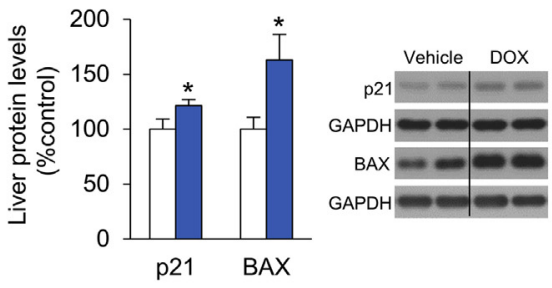

$\mathbf{F}$
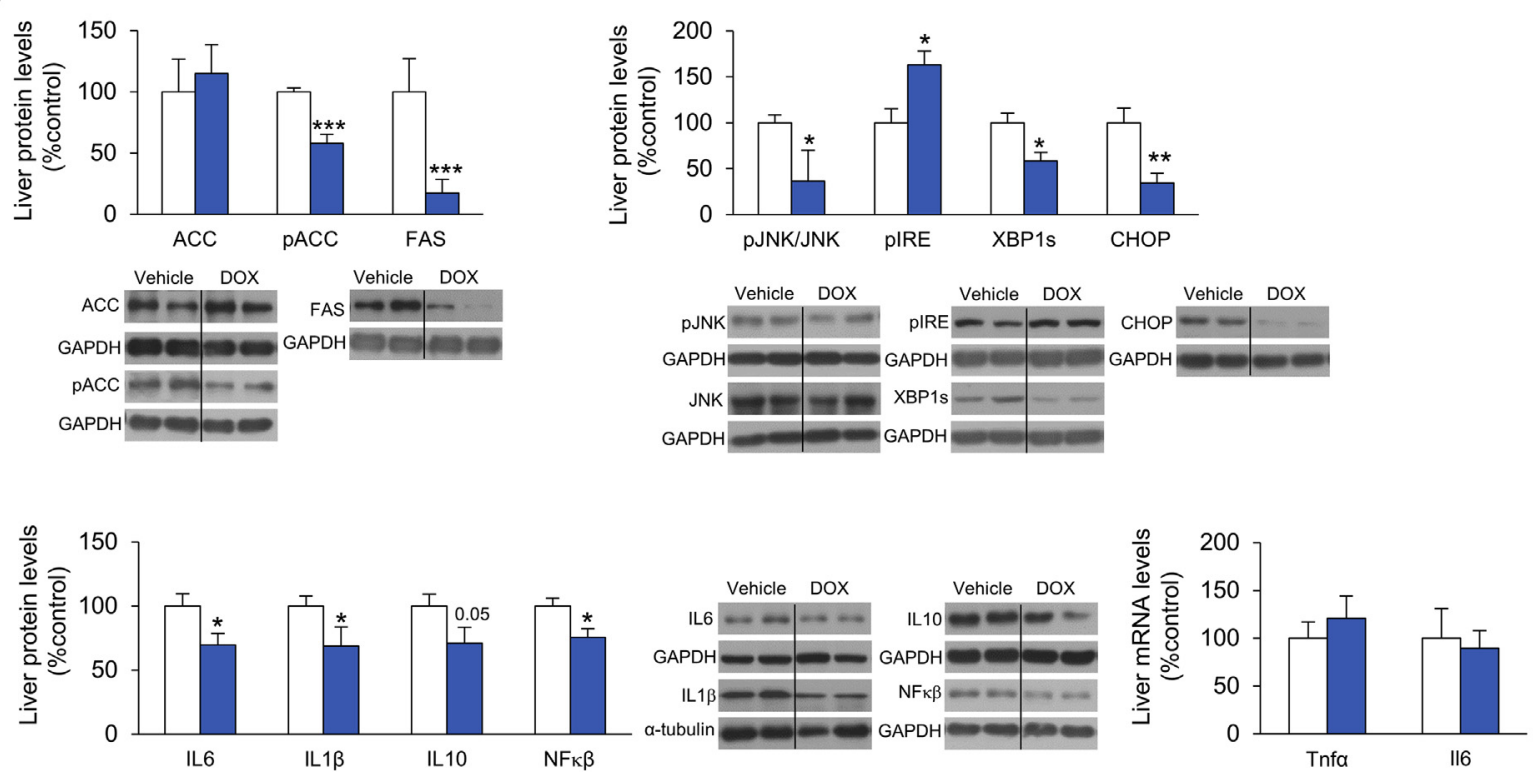

Figure 3: Doxorubicin treatment ameliorates MCD diet-induced liver NASH and ER stress. C57BL/6J mice fed with MCD diet for 2 weeks were randomly treated with either water or doxorubicin $(20 \mathrm{mg} / \mathrm{kg})$ co-administrated orally with quercetin $(15 \mathrm{mg} / \mathrm{kg})$ for another 2 weeks twice a week maintaining MCD diet. Body weight change (A). Representative photomicrographs of hematoxylin\&eosin (upper panel) and oil red 0 staining (lower panel) of liver sections, hepatic TG content and serum levels of AST and ALT (B). Representative images of liver sections with Sirius red staining and collagen-positive quantification and mRNA expression of collagen III (C). Phosphorylated levels of p53 and Bax in control and doxorubicin treated mice (D). mRNA levels of genes related to fatty acid oxidation in liver samples (E). Liver protein levels of proteins involved in: lipid deposition, ER stress, and protein levels and gene expression of inflammatory markers (F). HPRT and GAPDH were used to normalize mRNA and protein levels. Dividing lines indicate splicings in the same gel. Values are mean \pm standard error of the mean of $7-10$ animals per group. ${ }^{\star} p<0.05,{ }^{\star \star} p<0.01,{ }^{\star \star \star} p<0.001$ versus control group. 
A

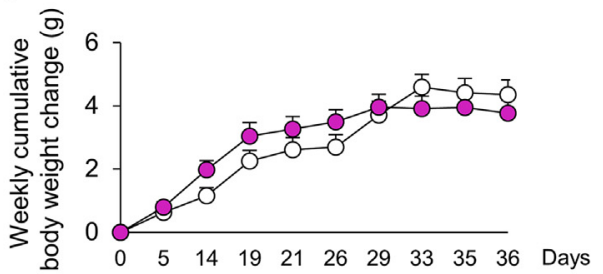

B

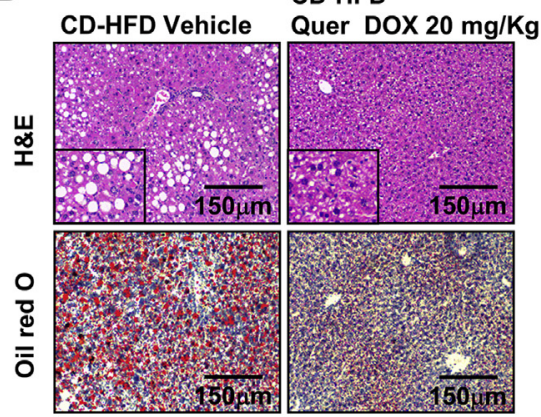

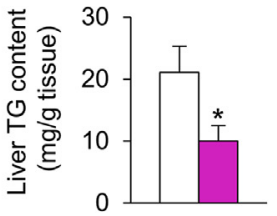

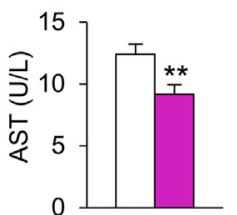

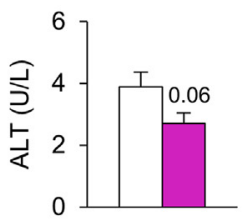

C
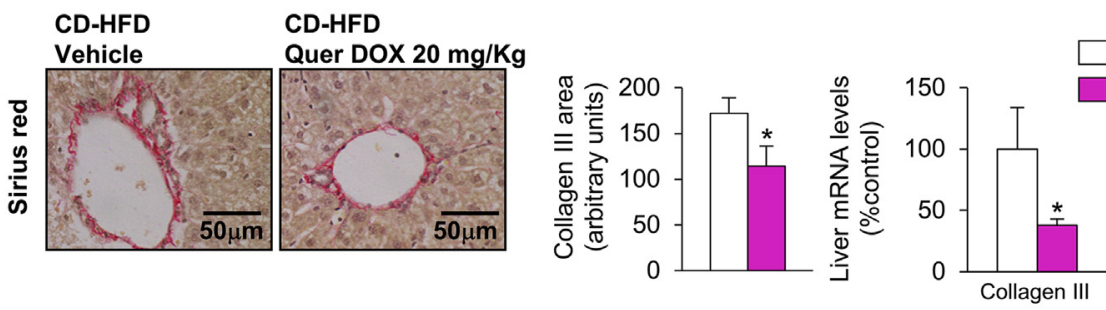

CD-HFD Vehicle

CD-HFD Quercetin $15 \mathrm{mg} / \mathrm{Kg}+$ DOX $20 \mathrm{mg} / \mathrm{Kg}$

D

E
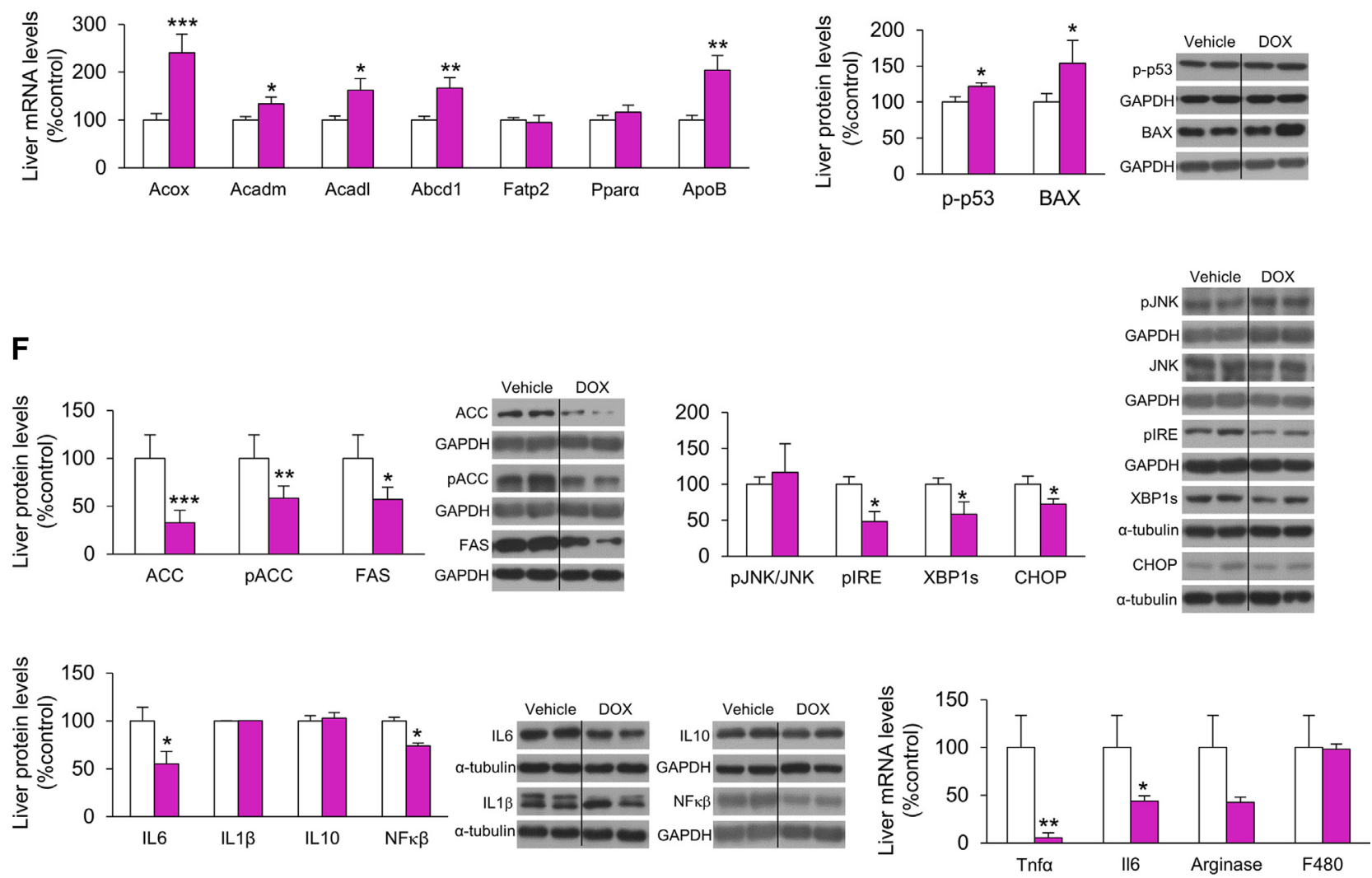

Figure 4: Doxorubicin treatment ameliorates choline deficient combined with high fat diet (CD-HFD)-induced liver NASH and ER stress. C57BL/6J mice fed a CD-HFD for 2 weeks were randomly treated with either water or doxorubicin $(20 \mathrm{mg} / \mathrm{kg})$ co-administrated orally with quercetin $(15 \mathrm{mg} / \mathrm{kg})$ for 2 weeks twice a week maintaining CD-HFD. Cumulative body weight (A). Representative photomicrographs of hematoxylin\&eosin (upper panel) and oil red 0 staining (lower panel) of liver sections, hepatic TG content and serum levels of AST and ALT (B). Representative images of liver sections with Sirius red staining and collagen-positive quantification and mRNA expression of collagen III (C). Phosphorylated levels of p53 and Bax in control and doxorubicin treated mice (D). mRNA levels of genes related to fatty acid oxidation in liver samples (E). Liver protein levels and gene expression of factors involved in: lipid deposition, ER stress, and inflammation (F). HPRT, tubulin and GAPDH were used to normalize mRNA and protein levels. Dividing lines indicate splicings in the same gel. Values are mean \pm standard error of the mean of $7-12$ animals per group. ${ }^{*} p<0.05,{ }^{* \star} p<0.01,{ }^{* * *} p<0.001$ versus control group. 
A

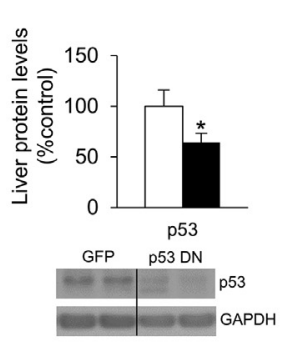

B

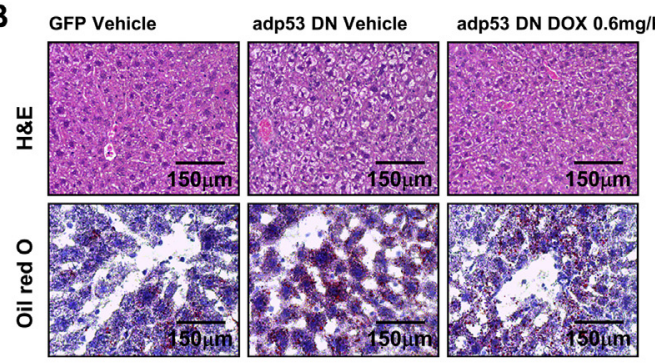

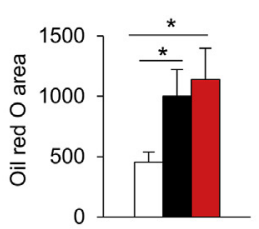

$\square$ adGFP + Vehicle

adp53 DN + Vehicle

adp53 DN + DOX 0.6mg/Kg

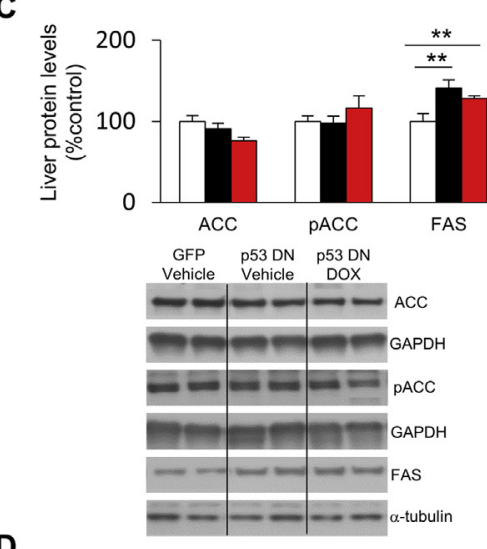

D

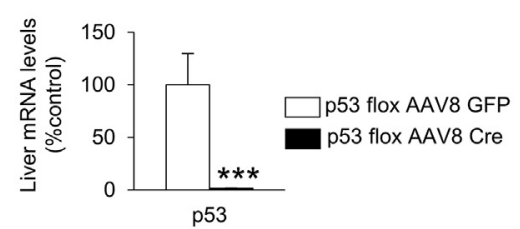

E
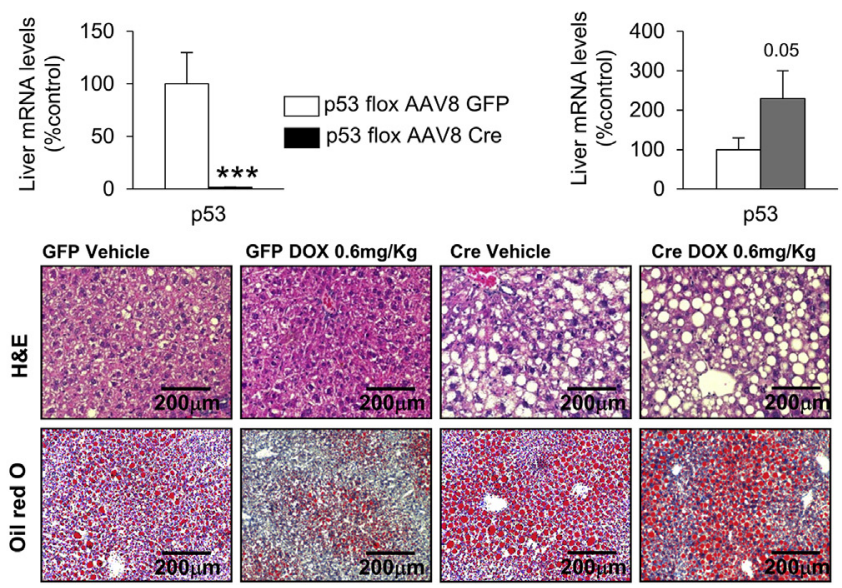

p53 flox AAV8 GFP + Vehicle

p53 flox AAV8 GFP + DOX $0.6 \mathrm{mg} / \mathrm{Kg}$

Vehicle vehicle p53DN

\begin{tabular}{c|c|c|c} 
& & pJNK \\
& & GAPDH
\end{tabular}

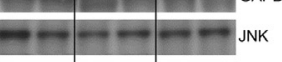

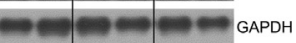

$-=-\infty$ PIRE

$=-m$ GAPDH
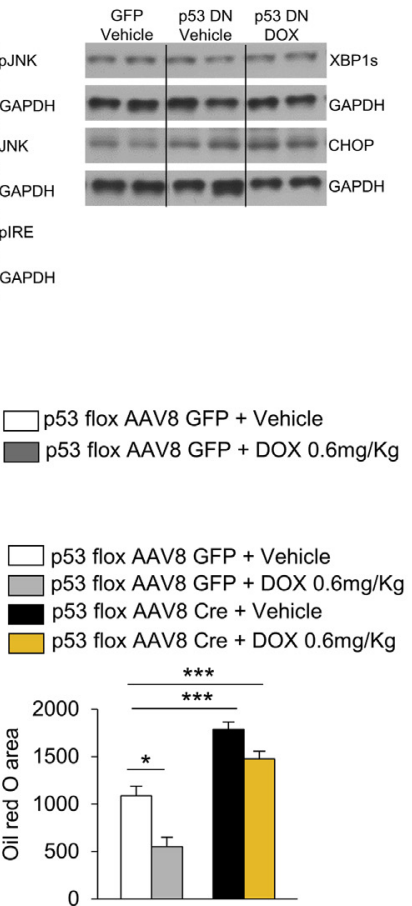

$\mathbf{F}$
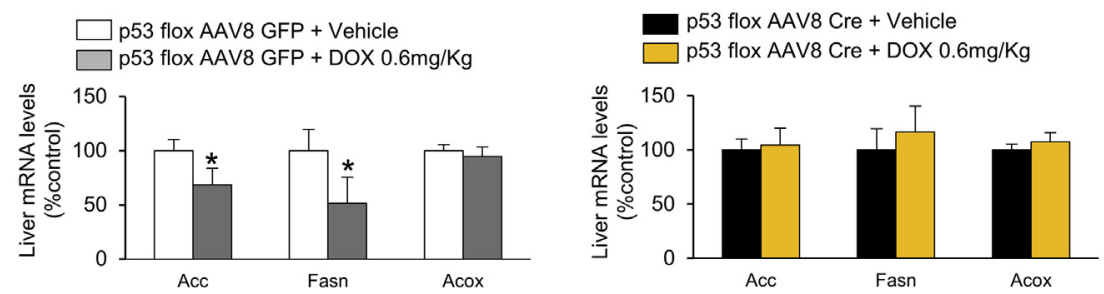

Figure 5: Doxorubicin treatment does not reverse lipid accumulation and ER stress in liver of mice with virogenetic down-regulation of p53. p53 protein levels in the liver after tail vein administration of adenoviral vectors encoding a green fluorescent protein (GFP) or a dominant negative form of p53 (adp53 DN) (A). Representative photomicrographs of hematoxylin\&eosin (upper panel) and oil red 0 staining (lower panel) of liver sections after tail vein administration of adenoviral vectors encoding a green fluorescent protein (GFP) or a dominant negative form of p53 (adp53 DN) (B). Liver protein levels of proteins involved in: lipid deposition and ER stress (C). p53 mRNA expression in the liver of p53 floxed mice fed a HFD injected in the tail vein with AAV8 encoding GFP or Cre recombinase (D). p53 mRNA expression in the liver of p53 floxed mice fed a HFD injected in the tail vein with AAV8 encoding GFP (D). Each group was then separated in two groups, one treated with saline i.p. and the other with doxorubicin $0.6 \mathrm{mg} / \mathrm{kg}$ twice per week for one month. Representative photomicrographs of hematoxylin\&eosin (upper panel) and oil red 0 staining (lower panel) of liver sections (E) and the red area quantification. Gene expression of factors involved in lipid deposition in the liver of p53 floxed mice fed a HFD injected in the tail vein with AAV8 encoding GFP or Cre recombinase (F). Tubulin and GAPDH were used to normalize protein levels. Dividing lines indicate splicings in the same gel. Values are mean \pm standard error of the mean of $7-9$ animals per group. ${ }^{\star} \mathrm{p}<0.05,{ }^{\star \star} \mathrm{p}<0.01,{ }^{\star \star \star} \mathrm{p}<0.001$ versus control group. 

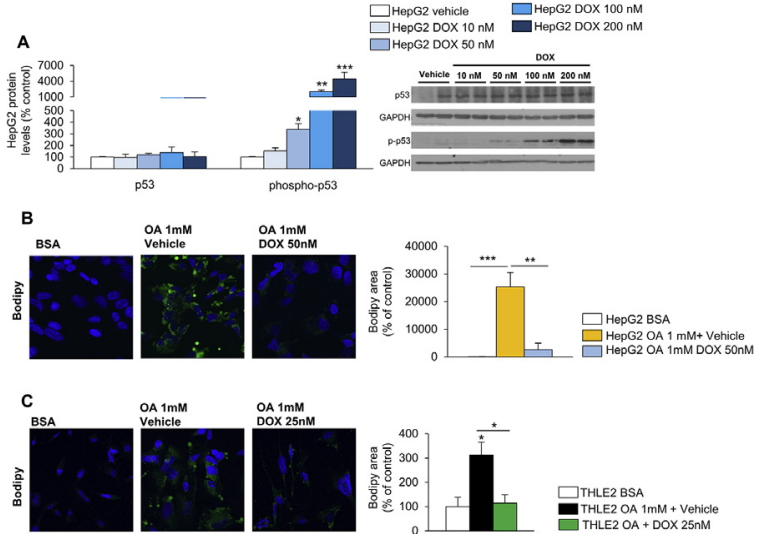

D
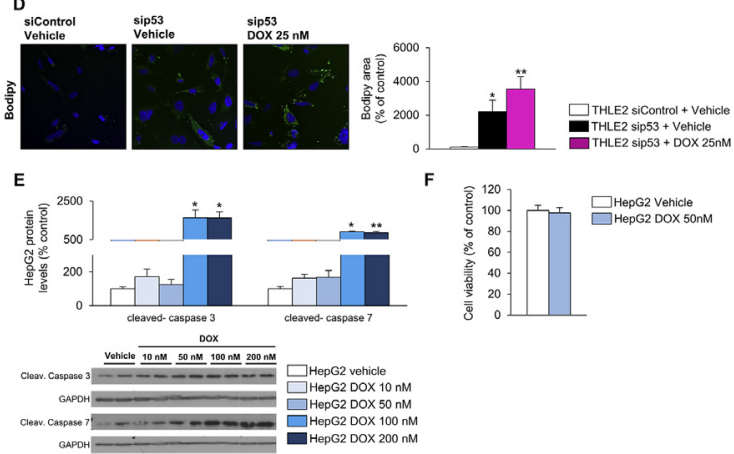

Figure 6: Effect of doxorubicin treatment in HepG2 and THLE2 cell lines. p53 and phosphorylated p53 protein levels after $48 \mathrm{~h}$ of a dose-response of doxorubicin in HepG2 cells (A). Representative dual channel fluorescent photomicrograph showing staining of lipids (BODIPY 493/503, green) and nuclei (DAPI, blue) in HepG2 cells cultured in oleic acid (1 mM) medium after treatment with doxorubicin $50 \mathrm{nM}$ for $48 \mathrm{~h}$ (B). Representative dual channel fluorescent photomicrograph showing staining of lipids (BODIPY 493/503, green) and nuclei (DAPI, blue) in THLE2 cells cultured in oleic acid (1 mM) medium after treatment with doxorubicin $25 \mathrm{nM}$ for $48 \mathrm{~h}(\mathrm{C})$. Representative dual channel fluorescent photomicrograph showing staining of lipids (BODIPY 493/503, green) and nuclei (DAPI, blue) in THLE2 cells after silencing p53 expression and then treated with doxorubicin $25 \mathrm{nM}$ for $48 \mathrm{~h}$ (D). Protein levels of cleaved caspase 3 and 7 after a dose-response experiment in HepG2 cells (E). Relative cell viability measured by MTT (3(4,5-dimethylthiazol-2-yl)-2,5-diphenyltetrazolium bromide) test comparing HepG2 cells treated with vehicle and doxorubicin $50 \mathrm{nM}$ during $24 \mathrm{~h}$ (F). GAPDH was used to normalize protein levels. Values are mean \pm standard error of the mean of 7-9 per group. ${ }^{\star} p<0.05,{ }^{\star \star} p<0.01,{ }^{\star \star \star} p<0.001$ versus control group.

was corroborated, and doxorubicin increased the expression of p53 in the liver of wild type mice (Figure 5D). Each group was then separated into two groups, one treated with vehicle i.p. and the other treated with doxorubicin $0.6 \mathrm{mg} / \mathrm{kg}$ i.p. Consistent with previous data, the lack of p53 in the liver caused a significant increase in the hepatic lipid content in control mice, but while doxorubicin was able to diminish the lipid content in control mice, it failed to do that in mice lacking hepatic p53 (Figure 5E). Additionally, the decreased expression of lipogenic genes such as Acc and Fas detected in normal mice treated with doxorubicin was not found in treated mice with depleted hepatic p53 (Figure 5F).

\subsection{Doxorubicin decreases lipid content in HepG2 and THLE2 cells independently of cell viability and apoptosis}

Once we demonstrated that doxorubicin regulates lipid metabolism in rodents via hepatic $\mathrm{p} 53$, we next assessed whether this drug could also exert the same effects in human hepatic cell lines. Thus, we challenged HepG2 cells with different doses of doxorubicin (10, 50, 100,200 , and $1000 \mathrm{nM}$ ) and found that the dose of $50 \mathrm{nM}$ was sufficient to phosphorylate p53 (Figure 6A). We next challenged HepG2 cells with oleic acid to induce lipid accumulation and then treated them with doxorubicin $50 \mathrm{nM}$, which caused a significant decrease in lipid content (Figure 6B). Similar results were observed in another human hepatic cell line, THLE-2 cells, in which oleic acidinduced lipid accumulation was also significantly decreased by doxorubicin (25 nM) (Figure 6C). Next, we performed an assay in THLE2 cells and silenced p53 using two different doses of sip53 (50 and $100 \mathrm{pmol})$. The results of this experiment show that p53 protein levels are markedly decreased after silencing p53 (Supplementary Figure 3A). In addition, silencing p53 in THLE2 significantly decreased the p53 downstream signaling as demonstrated by lower levels of p21 and Bax (Supplementary Figure 3B). Importantly, the effect of doxorubicin on lipid content did not occur when THLE-2 cells were transfected with siRNAs directed against p53 (Figure 6D), suggesting again that p53 is required for doxorubicin action on lipid metabolism.

Since p53 regulates cell viability and apoptosis, we next investigated whether the effects of doxorubicin on lipid metabolism were independent of its effects on cell cycle. As previously reported, we detected that when used a high dose, doxorubicin markedly activated p53 and apoptosis [13] (Figure 6E). However, the dose of $50 \mathrm{nM}$ did not affect the expression of cleaved caspase 3 or 7 (Figure 6E). Moreover, doxorubicin $50 \mathrm{nM}$ did not alter cell viability of HepG2 cells (Figure 6F). These results indicate that doxorubicin modulates lipid metabolism independent of its well-known effects on apoptosis or cell viability.

\subsection{Doxorubicin stimulates lipid oxidation and reduces de novo lipogenesis in HepG2 cells}

We next measured lipid oxidation and de novo lipogenesis directly in HepG2 cells treated with $50 \mathrm{nM}$ doxorubicin in the presence or absence of $1 \mathrm{mM}$ oleic acid. We found that doxorubicin up-regulated lipid oxidation, reflected as the measurement of palmitic acid oxidation (Figure 7B; complete oxidation, left; incomplete oxidation, right). Moreover, de novo TG and diacylglycerol lipogenesis and fatty acid synthesis were inhibited in HepG2 cells treated with doxorubicin (Figure 7A). The effect was more marked in the oleic acid treated HepG2 cells (Figure 7A). These results were confirmed when we measured the expression of genes involved in lipid oxidation in HepG2 cells challenged with doxorubicin $50 \mathrm{nM}$ for $48 \mathrm{~h}$. We found that doxorubicin stimulated the gene expression of Acox, Abcd1, and Fatp2 (Figure 7C).

\subsection{The actions of doxorubicin on lipid metabolism are independent of topoisomerase II}

Topoisomerase II (topo II) inhibitors are widely used in treatment protocols for hematologic malignancies and solid tumors. In a physiological setting, topoisomerase II is inhibited by p53 and it mediates many of the actions of p53. Thus, we investigated whether the effects of doxorubicin on hepatic lipid metabolism could be mediated by this enzyme. We measured protein levels of topoisomerase II in DIO mice treated with doxorubicin i.p. at 0.3 and $0.6 \mathrm{mg} / \mathrm{kg}$ for 2 months but failed to detect significant changes (Supplementary figure 4A). We also measured its protein levels after the oral administration of doxorubicin (20 mg/kg for one month) in mice fed a CD-HFD but again, were unable to see differences in comparison to vehicle treated mice (Supplementary Figure 4B). 
A
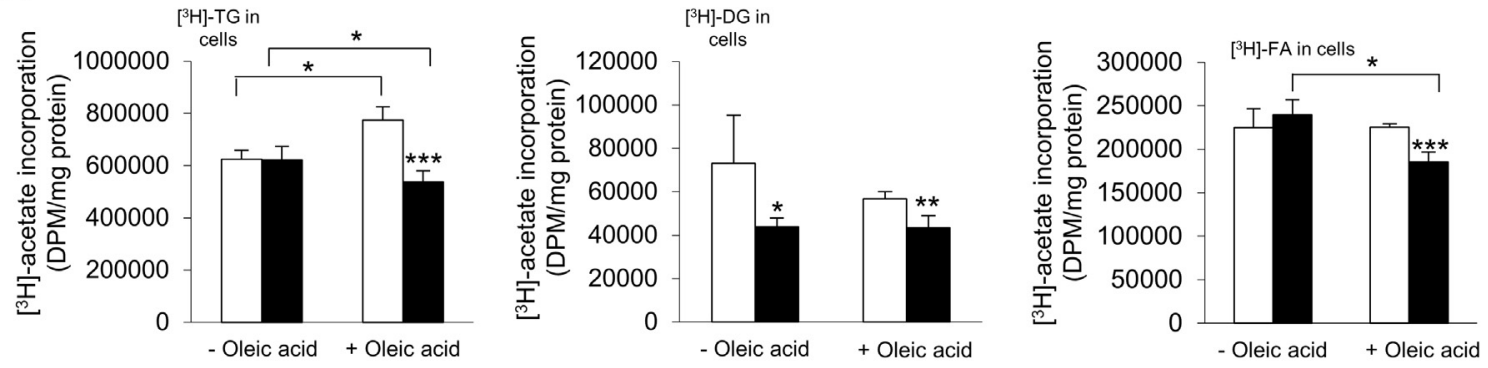

B
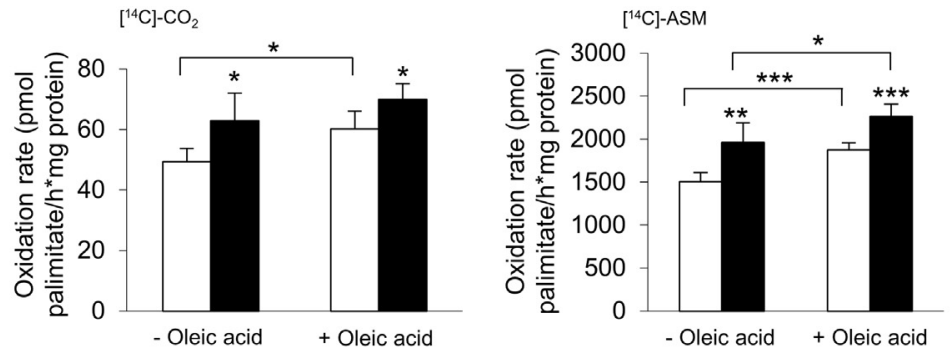

C

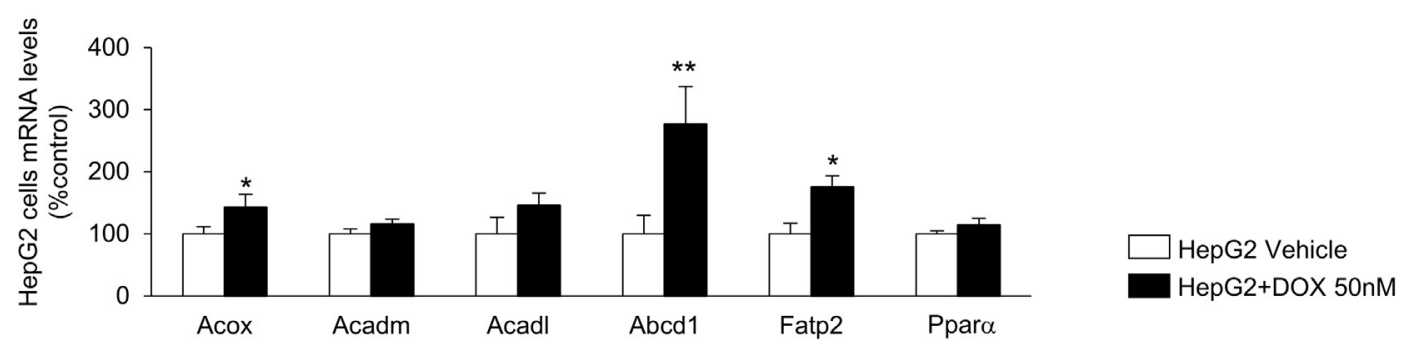

Figure 7: Doxorubicin treatment increases fatty acid oxidation and reduces de novo lipogenesis in HepG2 cells. De novo triglyceride (TG), diacylglycerol (DG) and fatty acid, phospholipid (FFA) (A). Complete and partial oxidation rate in HepG2 cells cultured in BSA and oleic acid medium after treatment with doxorubicin 50 nM (B). mRNA levels of genes related to fatty acid oxidation in HepG2 cells treated with doxorubicin $50 \mathrm{nM}(\mathrm{C})$. Values are mean \pm standard error of the mean of 5 per group. ${ }^{\star} \mathrm{p}<0.05$, ${ }^{\star \star} \mathrm{p}<0.01$, ${ }^{\star \star \star} \mathrm{p}<0.001$ versus control group.

\section{DISCUSSION}

The long-known Warburg hypothesis proposes that cancer cells predominantly produce energy by a high rate of glycolysis [14], which is essential to cope with the enormous energy requirements of metastatic growth. There is increasing evidence that the transcription factor $\mathrm{p} 53$, mostly known for its role as a tumor suppressor and its involvement in cellular homeostasis of cancer cells, is emerging as an important component in the regulation of metabolic homeostasis. Recent findings suggest that in addition to the high rate of glycolysis in cancer cells, reprogramming of lipid metabolism also occurs, since lipids are used for biosynthesis of membranes, post-translational modifications, second messengers for signal transduction, and as a source of energy during nutrient deprivation [15].

p53 generally functions as a negative regulator of lipid synthesis by activating fatty acid oxidation and inhibiting fatty acid synthesis [3], and loss of p53 activity and mutations in p53 accelerate lipid accretion. For instance, p53 induces lipin 1 , which ultimately regulates fatty acid oxidation in cultured cells [16]. Moreover, p53 is known to induce the expression of three carnitine acyltransferases (carnitine 0-octanoyltransferase, carnitine palmitoyltransferase $1 \mathrm{~A}$ and $1 \mathrm{C}[17]$ and induction of malonyl CoA decarboxylase [18], therefore promoting fatty acid oxidation. In addition to its role on fatty acid oxidation, p53 also inhibits lipogenesis by the inactivation of glucose-6-phosphate dehydrogenase [19] and the repression of sterol regulatory element-binding proteins [20]. Consistent with these results, our current pharmacological data indicate that the activation of p53 using low-doses of doxorubicin reduces the lipid accumulation in different experimental models of diet-induced NAFLD and NASH. Consistent with these findings, our results indicate that the activation of $\mathrm{p} 53$ stimulates fatty acid oxidation and decreases de novo lipogenesis. Moreover, the amelioration of NAFLD and NASH after the treatment with doxorubicin was accompanied by decreased expression of markers related to inflammation and ER stress. Thereby, our previous and current findings support a beneficial role for p53 on hepatocyte function in conditions of excessive lipid deposition. Other reports have hypothesized that $p 53$ is an essential player in the pathogenesis of NAFLD [21-24]. However, clear experimental evidence of liver-specific p53 as the driver of this process was shown only recently, demonstrating that when p53 was specifically inhibited in the liver there was a significant increase in lipid deposition $[4,5]$, while the hepatic rescue of p53 ameliorates liver steatosis in p53-null mice and mice fed a HFD [5]. 
A previous study also suggested a beneficial role for $\mathrm{p} 53$, demonstrating that diet-induced obese mice treated with the p53 inactivator pifithrin- $\alpha$ p-nitro attenuates steatosis and liver injury [21]. However, this compound is known to also have p53-independent actions $[25,26]$, and whether the hepatic effects exerted by pifithrin- $\alpha$ were specifically mediated via $\mathrm{p} 53$ was not tested. Our results indicate that the actions of doxorubicin in the liver require p53, because the effect was abolished in our experiments with liver-specific down-regulation of p53, using three different approaches: a) AAV8-mediated Cre-LoxP recombination in p53 floxed mice [5]; b) double-mutant kinase dead p53 regulating kinase acting as a dominant negative of p53 [8]; and c) silencing p53 in human hepatic cell lines, interventions that all blunted the effects of doxorubicin on lipid accumulation, inflammation. ER stress, fatty acid oxidation, and lipogenesis.

One of the main challenges of doxorubicin administration is its very low bioavailability. Therefore, to assess the possibility to use oral administration of doxorubicin in diet-induced NAFLD/NASH models, we combined it with quercetin, known to increase the bioavailability of orally administered doxorubicin [9]. This combination of drugs lead to beneficial effects comparable to when doxorubicin was administered i.p., with a similar decrease in hepatic lipid accumulation, diminished levels of markers of de novo fatty acid synthesis, inflammation, and ER stress as well as an up-regulation in fatty acid oxidation.

Although doxorubicin plays a key role in the regulation of cell cycle and apoptosis, our results indicate that at the doses of doxorubicin required to modulate lipid metabolism in the liver, cell proliferation and cell viability are unaffected. In this regard, topoisomerase II, an enzyme inhibited by $\mathrm{p} 53$ that mediates some of its actions, also remained unaltered at the doses of doxorubicin used here. Overall, our results indicate that the effects of doxorubicin on lipid metabolism can be completely dissociated from the "classical" actions of p53, which are effects that occur at much higher doses of doxorubicin than the ones required to act on fatty acid metabolism.

Finally, it is widely known that the use of doxorubicin as a chemotherapeutic agent (thereby given at high doses) has a dose-dependent off-target effects, including cytotoxicity on heart and other organs [27]. Thus, a key aspect of our results was to know if the doses used here to ameliorate diet-induced NAFLD/NASH were causing any cardiac alteration. Our results show that the levels of troponin, a biomarker for the detection of cardiac injury [28], remained unaltered between vehicle and doxorubicin-treated groups, and troponin was almost undetectable in comparison to a model of cardiac infarct. Thus, these data suggest that the i.p. and oral doses of doxorubicin efficient to ameliorate NAFLD and NASH were not causing any cardiac injury.

\section{CONCLUSIONS}

In summary, our results show that long-term pharmacological activation of p53 using i.p. or oral administration of doxorubicin at much lower doses than those used in oncology ameliorates liver injury. This finding was evident in two different dietary animal models of NAFLD and NASH; in mice fed a HFD or mice fed a MCD and choline deficient diet combined with HFD. The attenuation of liver injury was correlated with increased fatty acid oxidation, decreased de novo fatty acid synthesis, reduced inflammation, and lowered ER stress. We provide mechanistic insight evidence that these doxorubicin-mediated effects were dependent of $p 53$, since they were not observed in mice where hepatic p53 expression was missing or reduced. Finally, the effects of doxorubicin on lipid metabolism in the liver were not related to cell viability or apoptosis, and the doses of doxorubicin used did not cause any toxicity in the heart, either by the i.p. or the oral route. Overall, these results suggest that pharmacological activators of p53 may have beneficial effects in diet-induced NAFLD and NASH.

\section{ACKNOWLEDGEMENTS}

This work has been supported by grants from Ministerio de Economia y Competitividad (CD: BFU2014-55871; RN: BFU2015-70664-R; GS: SAF2016-79126-R; ML: SAF2015-71026-R; PA: SAF2015-64352-R), Xunta de Galicia (ML: 2015CP079; RN: 2015-CP080 and PIE13/00024), Helse Vest RHF (JF: Western Norway Regional Health Authority), Comunidad de Madrid (GS: S2010/BMD-2326); Govierno Vasco (PA: 2016-IT-336-10) and Fundación AstraZeneca (R.N.) Centro de Investigación Biomédica en Red (CIBER) de Fisiopatología de la Obesidad y Nutrición (CIBERobn). CIBERobn is an initiative of the Instituto de Salud Carlos III (ISCIII) of Spain which is supported by FEDER funds. The research leading to these results has also received funding from the European Community's Seventh Framework Programme under the following grant: RN: ERC StG-281408 and GS: ERC StG-260464.

\section{APPENDIX A. SUPPLEMENTARY DATA}

Supplementary data related to this article can be found at https://doi.org/10.1016/j. molmet.2017.12.005.

\section{CONFLICT OF INTEREST}

None declared.

\section{REFERENCES}

[1] Efeyan, A., Serrano, M., 2007. p53: guardian of the genome and policeman of the oncogenes. Cell Cycle 6:1006-1010.

[2] Vousden, K.H., Lane, D.P., 2007. p53 in health and disease. Nature Reviews Molecular Cell Biology 8:275-283.

[3] Goldstein, I., Rotter, V., 2012. Regulation of lipid metabolism by p53-fighting two villains with one sword. Trends in Endocrinology and Metabolism 23: $567-575$.

[4] Prokesch, A., Graef, F.A., Madl, T., Kahlhofer, J., Heidenreich, S., Schumann, A., et al., 2017. Liver p53 is stabilized upon starvation and required for amino acid catabolism and gluconeogenesis. The FASEB Journal 31:732-742.

[5] Porteiro, B., Fondevila, M.F., Delgado, T.C., Iglesias, C., Imbernon, M., Iruzubieta, P., et al., 2017. Hepatic p63 regulates steatosis via IKKbeta/ER stress. Nature Communications 8:15111.

[6] Berkers, C.R., Maddocks, O.D., Cheung, E.C., Mor, I., Vousden, K.H., 2013. Metabolic regulation by p53 family members. Cell Metabolism 18:617-633.

[7] Aas, T., Borresen, A.L., Geisler, S., Smith-Sorensen, B., Johnsen, H., Varhaug, J.E., et al., 1996. Specific P53 mutations are associated with de novo resistance to doxorubicin in breast cancer patients. Nature Medicine 2:811-814.

[8] Al-Massadi, 0., Porteiro, B., Kuhlow, D., Köhler, M., Gonzalez-Rellan, M.J., Garcia-Lavandeira, M., et al., 2016. Pharmacological and genetic manipulation of p53 in brown fat at adult but not embryonic stages regulates thermogenesis and body weight in male mice. Endocrinology 157:2735-2749.

[9] Choi, J.S., Piao, Y.J., Kang, K.W., 2011. Effects of quercetin on the bioavailability of doxorubicin in rats: role of CYP3A4 and P-gp inhibition by quercetin. Archives of Pharmacal Research 34:607-613.

[10] González-Terán, B., Matesanz, N., Nikolic, I., Verdugo, M.A., Sreeramkumar, V., Hernández-Cosido, L., et al., 2016. p38gamma and p38delta reprogram liver metabolism by modulating neutrophil infiltration. The EMB0 Journal 35:536-552. 
[11] Imbernon, M., Sanchez-Rebordelo, E., Romero-Picó, A., Kalló, I., Chee, M.J., Porteiro, B., et al., 2016. Hypothalamic kappa opioid receptor mediates both diet-induced and melanin concentrating hormone-induced liver damage through inflammation and endoplasmic reticulum stress. Hepatology 64: $1086-1104$.

[12] Wolf, M.J., Adili, A., Piotrowitz, K., Abdullah, Z., Boege, Y., Stemmer, K., et al., 2014. Metabolic activation of intrahepatic CD8 + T cells and NKT cells causes nonalcoholic steatohepatitis and liver cancer via cross-talk with hepatocytes. Cancer Cell 26:549-564.

[13] Wang, S., Konorev, E.A., Kotamraju, S., Joseph, J., Kalivendi, S., Kalyanaraman, B., 2004. Doxorubicin induces apoptosis in normal and tumor cells via distinctly different mechanisms. intermediacy of $\mathrm{H}(2) 0(2)$ - and p53dependent pathways. Journal of Biological Chemistry 279:25535-25543.

[14] Warburg, 0., Wind, F., Negelein, E., 1927. The metabolism of tumors in the body. The Journal of General Physiology 8:519-530.

[15] Santos, C.R., Schulze, A., 2012. Lipid metabolism in cancer. FEBS Journal 279:2610-2623.

[16] Assaily, W., Rubinger, D.A., Wheaton, K., Lin, Y., Ma, W., Xuan, W., et al., 2011. ROS-mediated p53 induction of Lpin1 regulates fatty acid oxidation in response to nutritional stress. Molecules and Cells 44:491-501.

[17] Goldstein, I., Ezra, 0., Rivlin, N., Molchadsky, A., Madar, S., Goldfinger, N., et al., 2012. p53, a novel regulator of lipid metabolism pathways. Journal of Hepatology 56:656-662.

[18] Liu, Yong, He, Yizhou, Jin, Aiwen, Tikunov, Andrey P., Zhou, Lishi, Tollini, Laura A., et al., 2014. Ribosomal protein-Mdm2-p53 pathway coordinates nutrient stress with lipid metabolism by regulating MCD and promoting fatty acid oxidation. Proc Natl Acad Sci U S A 111:E2414-E2422.

[19] Jiang, P., Du, W., Wang, X., Mancuso, A., Gao, X., Wu, M., et al., 2011. p53 regulates biosynthesis through direct inactivation of glucose-6-phosphate dehydrogenase. Nature Cell Biology 13:310-316.
[20] Yahagi, N., Shimano, H., Matsuzaka, T., Najima, Y., Sekiya, M., Nakagawa, Y., et al., 2003. p53 Activation in adipocytes of obese mice. Journal of Biological Chemistry 278:25395-25400.

[21] Derdak, Z., Villegas, K.A., Harb, R., Wu, A.M., Sousa, A., Wands, J.R., 2013. Inhibition of p53 attenuates steatosis and liver injury in a mouse model of nonalcoholic fatty liver disease. Journal of Hepatology 58:785-791.

[22] Yahagi, N., Shimano, H., Matsuzaka, T., Sekiya, M., Najima, Y., Okazaki, S., et al., 2004. p53 involvement in the pathogenesis of fatty liver disease. Journal of Biological Chemistry 279:20571-20575.

[23] Farrell, G.C., Larter, C.Z., Hou, J.Y., Zhang, R.H., Yeh, M.M., Williams, J., et al., 2009. Apoptosis in experimental NASH is associated with p53 activation and TRAIL receptor expression. Journal of Gastroenterology and Hepatology 24: $443-452$.

[24] Tomita, K., Teratani, T., Suzuki, T., Oshikawa, T., Yokoyama, H., Shimamura, K., et al., 2012. p53/p66Shc-mediated signaling contributes to the progression of non-alcoholic steatohepatitis in humans and mice. Journal of Hepatology 57:837-843.

[25] Kanno, S., Kurauchi, K., Tomizawa, A., Yomogida, S., Ishikawa, M., 2015. Pifithrin-alpha has a p53-independent cytoprotective effect on docosahexaenoic acid-induced cytotoxicity in human hepatocellular carcinoma HepG2 cells. Toxicology Letters 232:393-402.

[26] Sohn, D., Graupner, V., Neise, D., Essmann, F., Schulze-0sthoff, K., Janicke, R.U., 2009. Pifithrin-alpha protects against DNA damage-induced apoptosis downstream of mitochondria independent of p53. Cell Death \& Differentiation 16:869-878.

[27] Chatterjee, K., Zhang, J., Honbo, N., Karliner, J.S., 2010. Doxorubicin cardiomyopathy. Cardiology 115:155-162.

[28] Babuin, L., Jaffe, A.S., 2005. Troponin: the biomarker of choice for the detection of cardiac injury. CMAJ 173:1191-1202. 